\title{
Yield stress after double strain-path change
}

\author{
M.F. Vieira ${ }^{a, *}$, J.V. Fernandes ${ }^{b}$, B. Chaparro ${ }^{b}$ \\ a Departamento de Engenharia Metalúrgica e Materiais, GMM/IMAT, Faculdade de Engenharia da Universidade do Porto, Rua dos Bragas, P - \\ 4050-123 Porto, Portugal \\ ${ }^{\mathrm{b}}$ Departamento de Engenharia Mecânica, CEMUC, Faculdade de Ciências e Tecnologia da Universidade de Coimbra, \\ Polo II da Universidade de Coimbra - Pinhal de Marrocos, P - 3030 Coimbra, Portugal
}

Received 23 February 1999; received in revised form 3 February 2000

\begin{abstract}
Two different types of complex loading paths, with three strain paths (rolling-rolling-tension), were carried on a polycrystalline copper sheet. The first and second paths in rolling were performed perpendicular to each other. Depending on the case, the last strain path in tension was considered to be parallel to the first or second rolling path. The effect of double strain path change on the subsequent reloading yield stress is studied in this work. The results show that the behavior after prestraining depends mainly on the orientation relationship between the previous (rolling-rolling) and the subsequent (tension) paths and less on the order in which they have been performed. The mechanical behavior in reloading is discussed on the basis of a statistical study of the slip systems that remain active after path change. (C) 2000 Elsevier Science S.A. All rights reserved.
\end{abstract}

Keywords: Strain path change; Slip systems; Mechanical behavior

\section{Introduction}

When dealing with complex strain paths, it is often observed that the active slip systems change partially or totally after reloading. Thus, the mechanical behavior in the second strain path is characterised by a high yield stress, defined as the back extrapolated stress $\sigma_{\text {be }}$ (compared with the stress $\sigma_{\mathrm{r}}$ during the monotonic path at the same strain), which is followed by a relatively low work-hardening rate [1-7]. This behavior is mainly controlled by the magnitude of the strain path change, which is usually defined by a parameter $\alpha$, initially proposed by Schmitt et al. [8], that corresponds to the cosine of the angle between the two vectors that represent successive strain tensors.

Previous results [9-15] show that, in the case of sequences of two strain paths, the evolution of the reloading stress, with respect to the amplitude of the change of strain path, is related to the activation of new slip systems, which are typical of the reloading path. In polycrystalline copper, the average number of active

\footnotetext{
* Corresponding author. Tel.: + 351-22-2041785; fax: + 351-222041792.

E-mail address: mvieira@fe.up.pt (M.F. Vieira)
}

slip systems in the grains increases when $\alpha$ evolves from 1 to 0 . In fact, for $\alpha=1$, there is no change of strain path and the previously active slip systems are still active after reloading. Consequently, no significant changes in the mechanical behavior are detected $\left(\sigma_{\text {be }} /\right.$ $\left.\sigma_{\mathrm{r}}=1\right)$. In contrast, for $\alpha=0$ the strain path change is drastic, i.e. most of the active slip systems during reloading have not been activated during the first path. Therefore, latent hardening effects become significant and $\sigma_{\mathrm{be}} / \sigma_{\mathrm{r}}$ can take values close to 1.15 .

The relatively low work-hardening rate, observed in the early stage of reloading, is mainly due to the dynamic recovery of the previous dislocation structure, which is stronger when active slip systems are modified. The dislocation structure becomes unstable, when the deformation conditions change: the passage of groups of dislocations into dislocation tangles and cells tends to change their organisation and promote the annihilation of dislocations $[6,10,14,16]$. The previous dislocation structure is gradually replaced by another structure, typical of the new path. This structure has greater stability under the new loading conditions and, as a result, the mechanical behavior tends to the one typical of the current path. The intensity of dynamic recovery depends on the magnitude of the strain path 
change: for the same prestrain value, its intensity increases when the parameter $\alpha$ approaches zero.

In this paper, the hardening effects associated with the change of strain path on polycrystalline copper sheets are studied for sequences of three strain paths. The reloading yield stress in the third path, always in tension, is studied as a function of the history of deformation, under the two prestrain paths. In the context mentioned above, the results described allow us to understand how the memory of the first path prevails during a second path of the same type, both in rolling. This is possible by studying mechanical behavior during reloading, in a third path in tension.

\section{Experimental procedure}

Oxygen-free high purity copper $(99.95 \% \mathrm{Cu})$ sheet, 1-mm thick, previously cold-rolled and annealed, was used in this research. The grains were equiaxed with a small grain size $(7 \mu \mathrm{m})$.

\begin{tabular}{|c|c|c|c|}
\hline \multicolumn{4}{|c|}{ Sequences studied } \\
\hline \multicolumn{2}{|r|}{ RyRxTy } & \multicolumn{2}{|l|}{ RxRyTy } \\
\hline \multirow{7}{*}{$\alpha=0.87$} & $\begin{array}{c}\text { Ry - Rolling } \\
\text { (parallel to tension) }\end{array}$ & $\begin{array}{c}\mathrm{Rx}-\text { Rolling } \\
\text { (normal to tension) }\end{array}$ & \multirow{7}{*}{$\alpha=0$} \\
\hline & $\frac{1}{\alpha=0.5}$ & $\alpha=0.5$ & \\
\hline & $\downarrow$ & 1 & \\
\hline & $\begin{array}{c}\mathrm{Rx} \text { - Rolling } \\
\text { (normal to tension) }\end{array}$ & $\begin{array}{c}\text { Ry - Rolling } \\
\text { (parallel to tension) }\end{array}$ & \\
\hline & $\frac{\nabla}{\alpha=0}$ & $\stackrel{b}{\alpha=0.87}$ & \\
\hline & 1 & 7 & \\
\hline & Ty - Tension & Ty - Tension & \\
\hline
\end{tabular}

Fig. 1. Values of the parameter $\alpha$ that characterise the changes of strain path in the studied sequences.

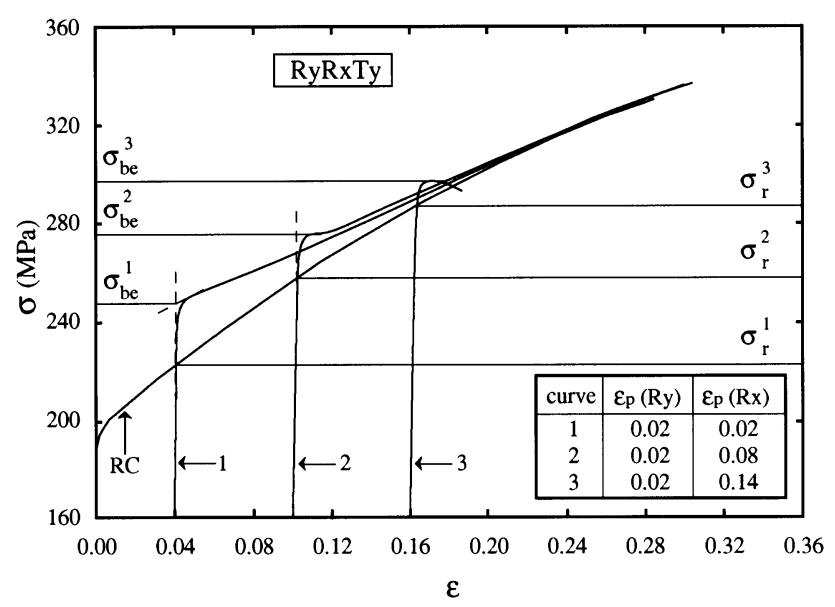

Fig. 2. Examples of true stress $(\sigma)$-true strain $(\varepsilon)$ curves in tension of prestrained samples showing three different types of behavior (see text for details).
X-ray analysis [17] was performed on samples without deformation and deformed up to 0.20 in rolling (normal and parallel to the previous rolling direction of the copper sheet). The initial texture of the sheet is quite smooth, nevertheless, it is possible to separate minor texture components such as: $\{110\}<1 \overline{1} 0>$, $\{110\}<1 \overline{1} 1>, \quad\{110\}<1 \overline{1} 2>, \quad\{112\}<1 \overline{1} 0>\quad$ and $\{123\}<3 \overline{3} 1>$. These results agree with the mean value of the $r$ factor determined experimentally, which is close to one $(r=0.98)$. No significant changes are detected in the texture during prestrain in rolling, up to 0.20 , which is a behavior usually observed in annealed copper sheet $[10,17,18]$.

Two types of sequences of strain paths rollingrolling-tension were performed:

1. RxRyTy: sequences of two normal rollings (RxRy) followed by uniaxial tension tests (Ty) performed on samples cut parallel to the second rolling direction (Ry);

2. RyRxTy: sequences of two normal rollings (RyRx) followed by uniaxial tension tests (Ty) performed on samples cut parallel to the first rolling direction (Ry).

The rolling direction $\mathrm{Rx}$ is parallel to the initial rolling direction of the sheet; the tensile direction $\mathrm{Ty}$ and the rolling direction $\mathrm{Ry}$ are parallel to the transverse direction of the sheet. The corresponding values of the strain path change parameter, defined in the last section, are indicated in Fig. 1. The studied cases concern the following values for $\alpha$ : $0,0.5$ and 0.87 .

The sequences of the three strain paths were performed at room temperature and at a von Mises strain rate of $\sim 7 \times 10^{-4} \mathrm{~s}^{-1}$. Square samples of $10^{4} \mathrm{~mm}^{2}$ were used for rolling. In both sequences studied, RxRyTy and RyRxTy, the total von Mises equivalent prestrain value during the two first paths in rolling, was within the range $0-0.20$. The strain values used in the second rolling were $0.02,0.04,0.06,0.08$ and 0.10 . The third path in tension was performed on samples that had gauge length dimensions of $60 \times 10 \mathrm{~mm}$. A $50-\mathrm{mm}$ strain gauge was used to measure the extension.

\section{Results}

The mechanical behavior of prestrained samples is presented in Fig. 2. In this figure, the reloading yield stresses (defined as $\sigma_{\text {be }}$ ) during the last path in tension, is defined for three distinct cases. After low total prestrain values, the hardening curve tends towards the monotonic one, presenting a significantly residual uniform strain. The hardening curve drops immediately after yielding, for high total prestrain values. In this case, the residual uniform strain is almost equal to zero. For moderate total prestrain values (at $\sim 0.06-0.10$, depending on the cases), the stress-strain curve can 


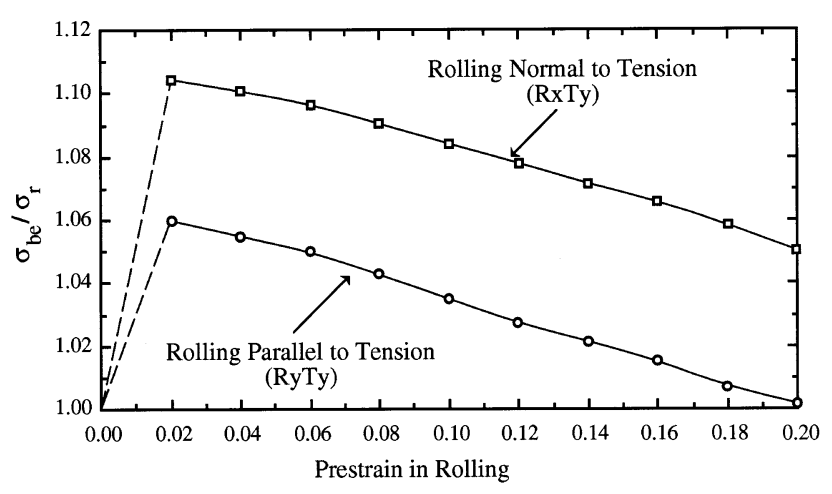

Fig. 3. Evolution of $\sigma_{\mathrm{be}} / \sigma_{\mathrm{r}}$ with the prestrain value for the sequences: rolling parallel (RyTy) and normal (RxTy) to the last path in tension.
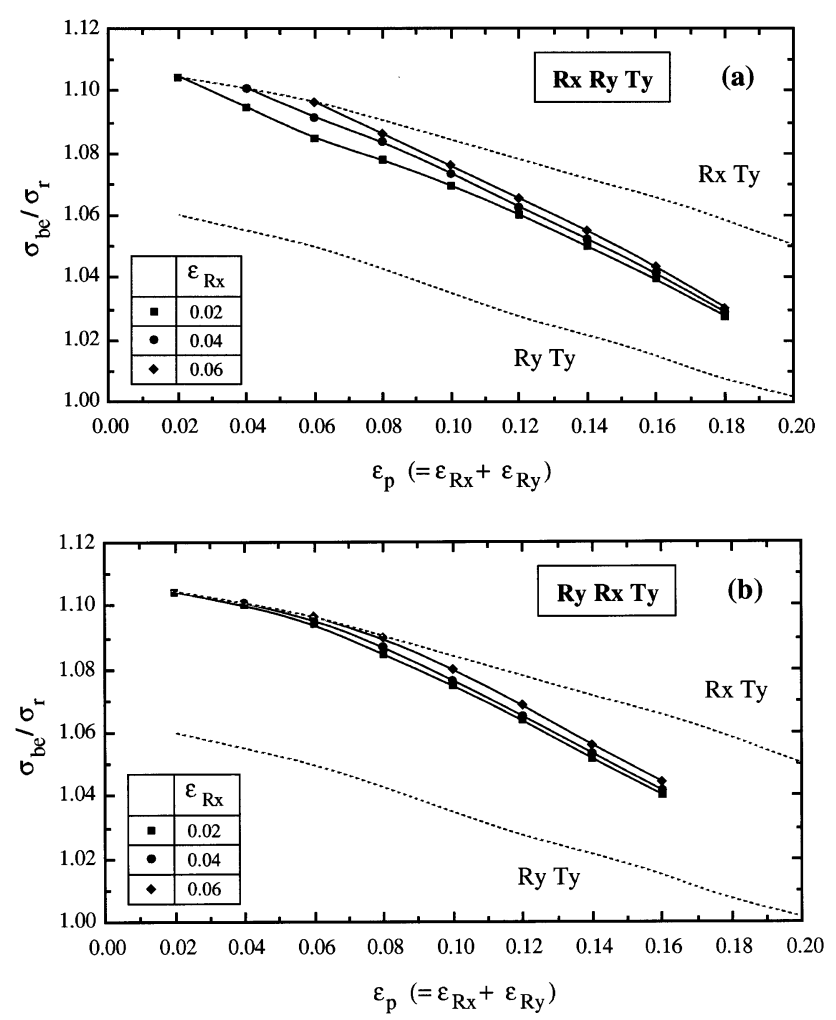

Fig. 4. Evolution of $\sigma_{\mathrm{be}} / \sigma_{\mathrm{r}}$ with the total prestrain value during the two preloading rolling paths: (a) sequence RyRxTy and (b) sequence RxRyTy. The prestrain values of rolling normal to tension are 0.02 , 0.04 and 0.06 . The cases of the sequences RyTy and RxTy (Fig. 3) are also shown for comparison purposes.

present a low work-hardening rate, at the beginning of deformation, followed by an increase in slope, at high strain. The fact that the slope of the true stress-strain curve is always greater than zero means that deformation is not of the Lüders band type.

In order to understand the behavior during the sequences of the three strain paths, the cases of simple rolling followed by tension are presented first. Then, the behavior during sequences of rolling-tension, with rolling parallel and normal to the tensile axis, is introduced. In Fig. 3, the values $\sigma_{\text {be }} / \sigma_{\mathrm{r}}$ are shown as a function of the prestrain value in rolling. For the same prestrain value, the values of $\sigma_{\mathrm{be}} / \sigma_{\mathrm{r}}$ are higher in the case of rolling normal to tension RxTy than in the case of rolling parallel to tension RyTy, which is consistent with previous results on copper $[19,20]$.

Fig. 3 shows that the value of $\sigma_{\mathrm{be}} / \sigma_{\mathrm{r}}$ decreases with the prestrain value, for both RxTy and RyTy. Considering that the crystallographic texture does not evolve, the explanation for this effect is certainly related to the definition used for the equivalent prestrain. In fact, the position of the origin of the reloading curve, and consequently the value of $\sigma_{\mathrm{be}} / \sigma_{\mathrm{r}}$, depends on the definition of the rolling equivalent prestrain. Different ways can be used to define the equivalent strain. The von Mises strain is often used, as is the case in the present work. Sometimes, the equivalent strain is a function of the plastic work: two strain values are equivalent when the plastic work performed during the corresponding paths is equal for both cases. This criterion has already been used to calculate $\sigma_{\text {be }} / \sigma_{\mathrm{r}}$ for sequential tension tests in copper [10]. Both methods give fairly close results, although the latter seems to be the most satisfactory. For the present case, it is not possible to use the last criterion because measurements of the load as a function of the strain are not possible during rolling. Moreover, in the context of this work, the use of the von Mises equivalent strain is enough: distinct levels of the values of $\sigma_{\mathrm{be}} / \sigma_{\mathrm{r}}$ as a function of the prestrain were found for the paths RxTy and RyTy (Fig. 3).

In the case of the sequences of the three strain paths, the dependence of the normalised reloading yield stress on the total prestrain value in rolling (normal and parallel to tension) is shown in Fig. 4. This figure displays the evolution of $\sigma_{\mathrm{be}} / \sigma_{\mathrm{r}}$ for sequences of the three strain paths and for the cases of prestrain in rolling normal to tension equal to $0.02,0.04$ and 0.06 . The results for sequences of only two strain paths in rolling-tension (Fig. 3) are also shown in these figures. Fig. 4(a) considers the cases RxRyTy, for which rolling normal to tension is the first path. The behavior in the cases RyRxTy, for which rolling normal to tension is the last prestrain path, is shown in Fig. 4(b). For both sequences, the values of $\sigma_{\mathrm{be}} / \sigma_{\mathrm{r}}$ evolve with the total prestrain values in such a way that low total prestrain values approach the case of the sequence rolling normal to tension (RxTy) and high total prestrain values tend to approach the case of the sequence rolling parallel to tension (RyTy). This is observed independently of the prestrain value in rolling normal to tension, which has almost no influence on the behavior.

\section{Discussion}

The increase in yield stress after a path change, observed in the complex strain paths analysed in this 
work, can be related to the physical mechanisms that occur during reloading. The evolution of the crystallographic texture during prestrain in rolling does not explain this hardening: there is almost no evolution of the texture during prestrain in rolling, up to 0.20 , as mentioned above. Thus, the latent hardening effect plays an important role in this behavior, which can be analysed by investigating the nature of the active slip systems during the two successive strain paths [10,11]. A statistical analysis of the sets of active slip systems during each path was performed. In this analysis, 294 grain orientations were studied, assumed to be representative of all crystal orientations present in the polycrystal [21]. The mean results, corresponding to the three single strain path change (RyTy, RxRy and RxTy), show that:

- For RyTy $(\alpha=0.87), 70 \%$ of the slip activity in the second path (Ty) occurs in systems already active in the first path (Ry).
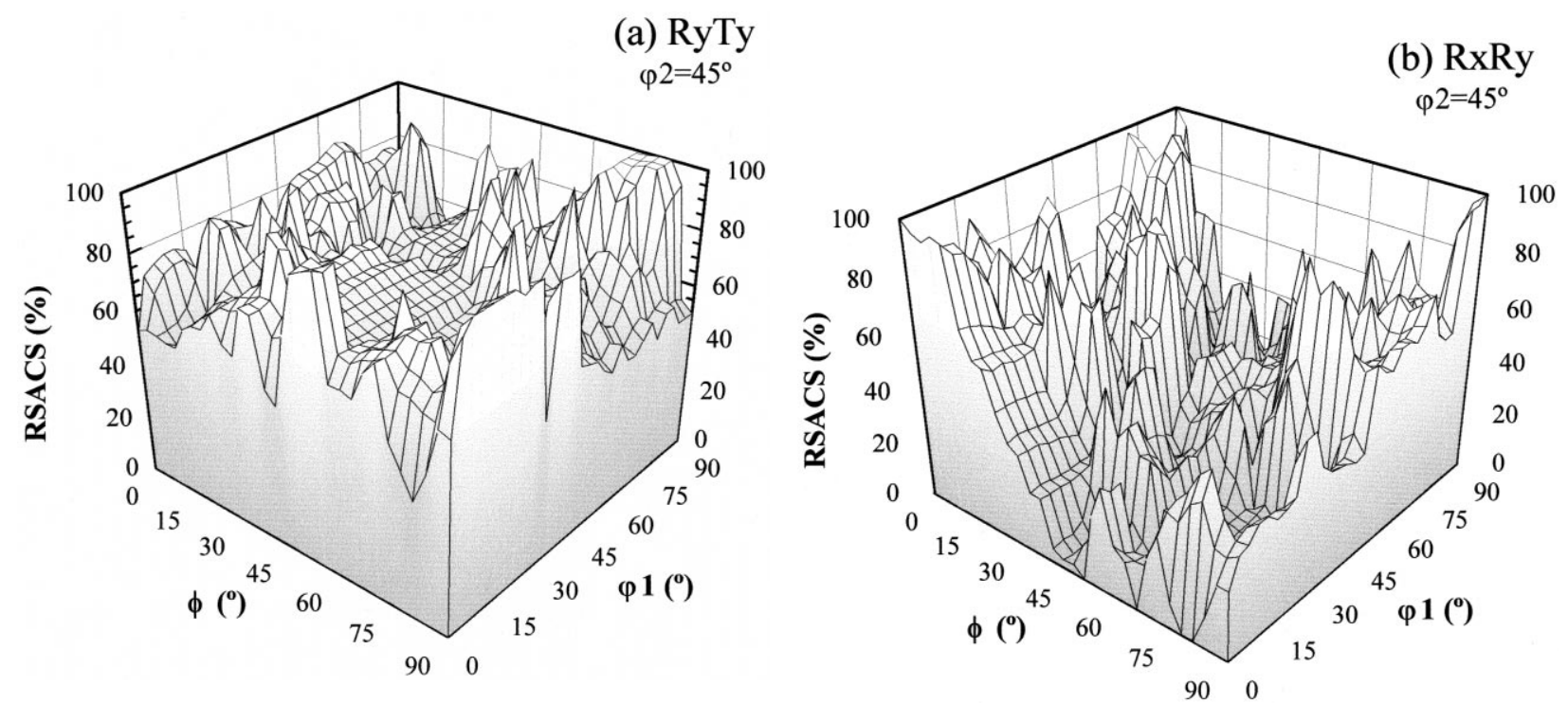

(c) RxTy

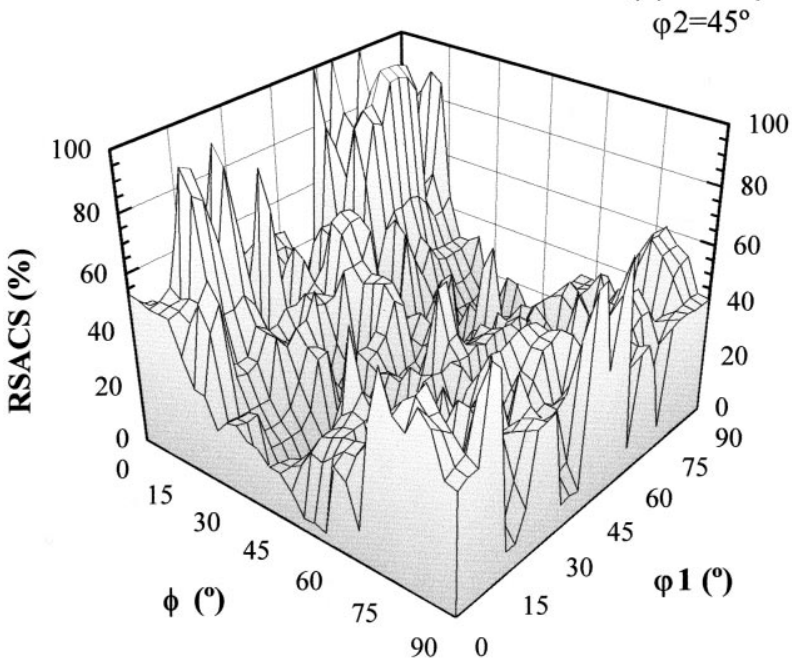

Fig. 5. Reloading slip activity in coincident systems (RSACS) as a function of grain orientation: (a) sequence RyTy, (b) sequence RxRy and (c) sequence RxTy. The grain orientations are described in the $\varphi_{2}=45^{\circ}$ Euler section.
- For RxRy or RyRx ( $\alpha=0.5)$, the importance of the new active slip systems is already meaningful. In fact, $\sim 60 \%$ of the slip activity in the second path (Ry or Rx) occurs in new active systems, i.e. systems that have been inactive in the first path (Rx or Ry).

- For RxTy $(\alpha=0)$, in most grains, the active slip systems during reloading were not activated during the first path. Less than $30 \%$ of the slip activity in the second path (Ty) occurs in systems that were active in the first path $(\mathrm{Rx})$. In this path change, generally, the occurrence of a new slip system corresponds to the slip of dislocations with a new Burgers vector, which was not activated during prestrain.

Moreover, some particular orientations were studied in detail as shown in Fig. 5. In this figure, the reloading slip activity in coincident systems (RSACS) is shown as a function of grain orientation. The Euler angles $\varphi_{1}, \varphi_{2}$ and $\Phi$ [22] were used to describe the grain orientations 


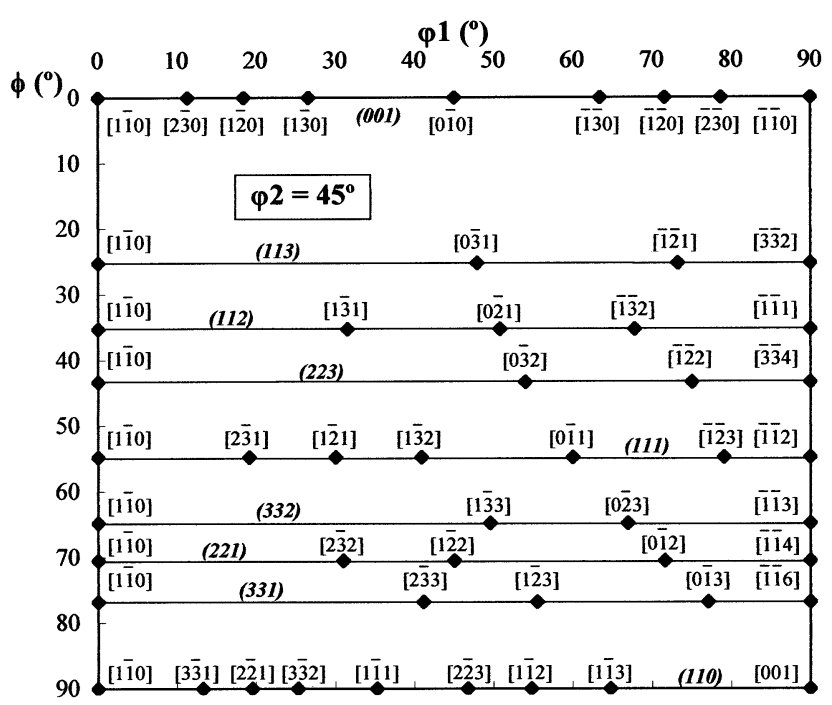

Fig. 6. Section of the Euler space $\left(\varphi_{2}=45^{\circ}\right)$ illustrating the correspondence between Euler angles $\left(\varphi_{1}, \varphi_{2}, \Phi\right)$ and Miller indices (hkl) [uvw].

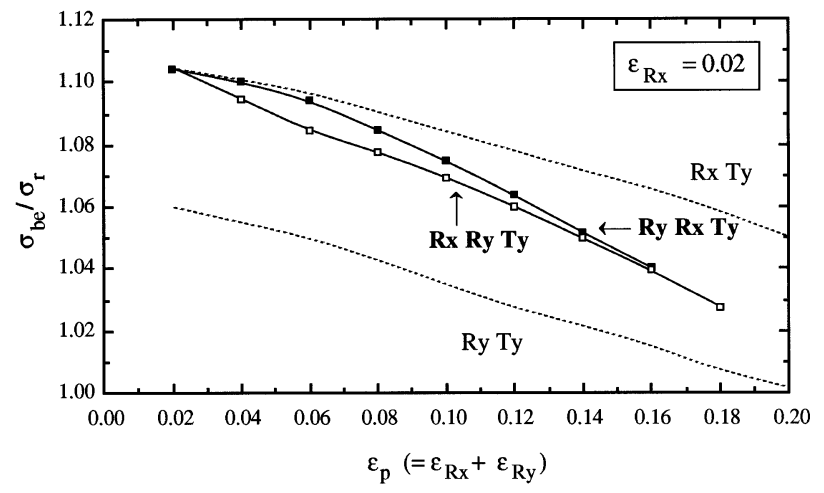

Fig. 7. Evolution of $\sigma_{\mathrm{be}} / \sigma_{\mathrm{r}}$ with total prestrain value during the two preloading rolling paths, for a prestrain value in the path rolling normal to tension ( $\mathrm{Rx}$ ) equal to 0.02 . Comparison between the sequences RyRxTy and RxRyTy.

with respect to the external axes of the sample. The calculations were made for grain orientations on a grid in Euler space where $\varphi_{1}$ and $\Phi$ were varied in $6^{\circ}$ steps and keeping $\varphi_{2}$ constant $\left(\varphi_{2}=45^{\circ}\right)$. The Euler angles can be transformed into the familiar description (hkl) [uvw]. Fig. 6 illustrates, for the Euler section used in Fig. 5, some of the corresponding Miller indices. This Euler section was chosen because it includes the texture components of the sheet. From the analysis of Fig. 5 it can be concluded that, for $\alpha=0.5$ and $\alpha=0$ (severe strain path change), the slip activity after path change is strongly influenced by the grain orientation, namely for $\alpha=0$, strong gradients are observed in the values of the coincident active slip systems.

These important differences in the evolution of the active slip systems after recharge explain the distinct mechanical behavior, for the values of $\sigma_{\mathrm{be}} / \sigma_{\mathrm{r}}$, observed for the RxTy $(\alpha=0)$ and RyTy $(\alpha=0.87)$ strain path changes.

For the sequence RxRyTy, the first path change is characterised by $\alpha=0.5$ and the second by $\alpha=0.87$ (Fig. 1). Both path changes are relatively smooth, particularly the second one. The results shown in Fig. 4 indicate that, in this case, the memory of the first rolling ( $\mathrm{Rx})$ is noticeable in the tension (Ty), even when a strong deformation value is imposed on the second rolling (Ry). In the case of the sequence RyRxTy, the first path change is of the same type $(\alpha=0.5)$ but the second one is 'harder' $(\alpha=0)$. In this case, the yield behavior during the third path in tension (Ty) is still more influenced by the presence of rolling normal $(\mathrm{Rx})$ to tension. This can be easily seen in Fig. 7 for the case of $\mathrm{Rx}=0.02$. In both sequences, RxRyTy or RyRxTy, the influence of the rolling normal to tension can still be clearly observed after 0.16 total prestrain $(\mathrm{Rx}+\mathrm{Ry})$, even when a strain value of only $\mathrm{Rx}=0.02$ is used.

These results show that the influence of the strain path on the mechanical behavior mostly depends on its orientation relationship with the subsequent paths. A 'hard' orientation between two strain paths (as already mentioned the hardest orientation corresponds to $\alpha=$ 0 ), whether consecutive or not imposes its effect on subsequent behavior. This means that the presence of an 'alien' dislocation microstructure, even as a result of a low strain value, is an important feature of the behavior in reloading. This importance arises from the immediate effects on the behavior (sequence RyRxTy) and from the difficulty of erasing them during deformation (sequence RxRyTy). Thus, the effects of an 'alien' microstructure prevail, even after two smooth changes of strain paths.

This is corroborated by the fact that a smooth strain path change does not significantly influence the subsequent behavior, as can be clearly seen in Fig. 8. This figure is as for Fig. 4, but for prestrain in rolling parallel to tension equal to $0.02,0.04$ and 0.06 . For sequences RxRyTy and RyRxTy, the values of $\sigma_{\mathrm{be}} / \sigma_{\mathrm{r}}$ quickly attain those of the sequence RxTy. This confirms that the behavior is mainly determined by any 'hard' change of strain path.

\section{Conclusions}

Hardening effects associated with the change of strain path on polycrystalline copper sheets were studied for two different types of sequences of three strain paths. The evolution of the normalised reloading yield stress with the total prestrain value, after two prestrain paths in rolling normal and parallel to the subsequent tensile axis, was analysed. It was found that the normalised reloading yield stress depends mainly on the 

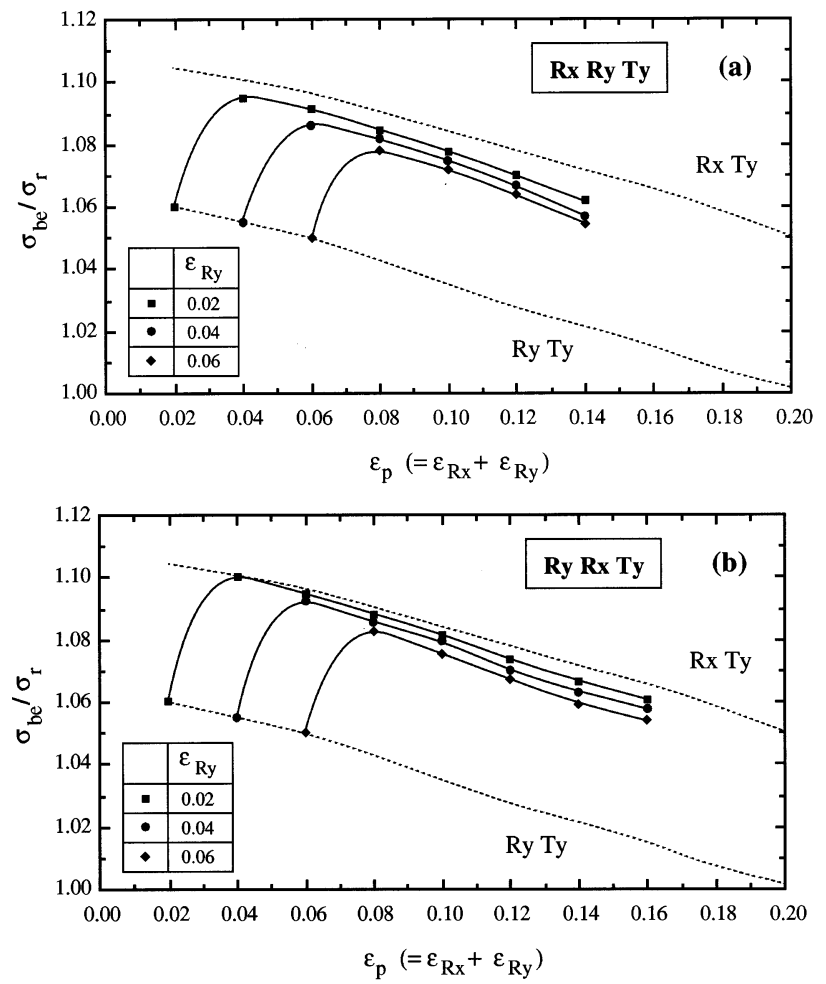

Fig. 8. Evolution of $\sigma_{\mathrm{be}} / \sigma_{\mathrm{r}}$ with the total prestrain value during the two preloading rolling paths: (a) sequence RyRxTy and (b) sequence RxRyTy. The prestrain values of rolling parallel to tension are 0.02 , 0.04 and 0.06 . The cases of the sequences RyTy and RxTy (Fig. 3) are also shown for comparison purposes.

orientation relationship between the previous and the subsequent path, and less on the order in which they have been performed. The main conclusion to be drawn is as follows: a 'hard' orientation between two strain paths, consecutive or not, determines the mechanical behavior in reloading.

\section{Acknowledgements}

The authors are indebted to Fundação para a Ciência e Tecnologia do Ministério da Ciência e Tecnologia and Program Praxis XXI, for financial support.

\section{References}

[1] R.H. Wagoner, J.V. Laukonis, Metall. Trans. 14A (1983) 1487.

[2] K. Chung, R.H. Wagoner, Metall. Trans. 17A (1986) 1001.

[3] M. Zandrahimi, S. Platias, D. Price, D. Barret, P.S. Bate, W.T. Roberts, D.V. Wilson, Metall. Trans. 20A (1989) 2471

[4] A.B. Doucet, R.H. Wagoner, Metall. Trans. 20A (1989) 1483.

[5] A.B. Doucet, S. Natarajan, Metall. Trans. 22A (1991) 393.

[6] P.S. Bate, Metall. Mater. Trans. 24A (1993) 2679.

[7] D.V. Wilson, P.S. Bate, Acta Metall. Mater. 42 (1994) 1099.

[8] J.-H. Schmitt, E. Aernoudt, B. Baudelet, Mater. Sci. Eng. 75 (1985) 13.

[9] J.L. Raphanel, E.F. Rauch, E.L. Shen, J.-H. Schmitt, Scripta Metall. 21 (1987) 1087.

[10] J.-H. Schmitt, J.V. Fernandes, J.J. Grácio, M.F. Vieira, Mater. Sci. Eng. A147 (1991) 143

[11] J.V. Fernandes, J.J. Grácio, J.-H. Schmitt, in: C. Teodosiu, J.L. Raphanel, F. Sidoroff (Eds.), Large Plastic Deformation: Fundamental Aspects and Applications to Metal Forming, Balkema, Rotterdam, 1993, p. 219

[12] E.F. Rauch, S. Thuillier, Mater. Sci. Eng. A164 (1993) 255.

[13] S. Thuillier, E.F. Rauch, Acta Metall. Mater. 42 (1994) 1973.

[14] J.V. Fernandes, J.H. Schmitt, Phil. Mag. A 48 (1983) 841.

[15] J.V. Fernandes, J.J. Grácio, J.-H. Schmitt, E.F. Rauch, Scripta Metall. Mater. 28 (1993) 1335.

[16] J. Washburn, G. Murty, Can. J. Phys. 45 (1967) 463.

[17] J.V. Fernandes, M.F. Vieira, Metall. Mater. Trans. 28A (1997) 1169

[18] J.J. Grácio, J.V. Fernandes, J.-H. Schmitt, Mater. Sci. Eng. A118 (1989) 97.

[19] M.F. Vieira, J.-H. Schmitt, J.J. Grácio, J.V. Fernandes, J. Mater. Process. Technol. 24 (1990) 313.

[20] M.F. Vieira, J.V. Fernandes, J. Mater. Process. Technol. 47 (1995) 261.

[21] J. Gil Sevillano, P. van Houtte, E. Aernoudt, Prog. Mater. Sci. 25 (1980) 69.

[22] H.J. Bunge, Mathematische Methoden der Texturanalyse, Akademie, Berlin, 1969. 